\title{
Correlação entre dietas restritivas e o surgimento ou agravamento de transtornos alimentares
}

\author{
Correlation between restrictive diets and the growth or aggravation of food disorders
}

\author{
Daniele Andreia Alvares $^{1^{*} \bullet}$, Aline Alves de Santana ${ }^{2} \bullet$ \\ ${ }^{1}$ Graduada em Nutrição da Universidade Nove de Julho, São Paulo, São Paulo, Brasil. ${ }^{2}$ Docente na Universidade Nove de Julho, São \\ Paulo, São Paulo, Brasil. *Autor para correspondência. E-mail: daniele_andreia@hotmail.com
}

\begin{abstract}
Resumo: Introdução: A obesidade é um problema de saúde pública, tornando hoje em dia a perda de peso uma apreensão comum, abrangendo quase todas as faixas etárias. Diante disto, surge a ideia de perda ponderal rápida, impactando no surgimento de dietas populares para erradicar a obesidade. Objetivo: demonstrar através de vários estudos, os reais motivos à prática desenfreada de dietas restritivas e sua repercussão negativa no desenvolvimento dos transtornos alimentares. Objetivo deste trabalho foi demonstrar através de vários estudos, os reais motivos à prática desenfreada de dietas restritivas e sua repercussão negativa no desenvolvimento dos transtornos alimentares. Revisão: Foi realizada uma revisão da literatura com o intuito de abordar a temática dietas da moda e os seus efeitos sobre a saúde dos seus seguidores. Discussão: O anseio pelo emagrecimento incrustado nas sociedades, é percebido pela literatura como um dos fatores que colaborariam para o crescimento desordenado destes transtornos na atualidade. Considerações finais: Nota-se que independentemente da motivação á pratica de dietas restritivas, estas adquirem uma conotação de tolhimento, impossibilitando uma qualidade de vida satisfatória.
\end{abstract}

Palavras-chave: dietas restritivas, dietas da moda, índice de alimentação saudável, análise de dietas da moda, transtornos alimentares.

\begin{abstract}
Introduction: Obesity is a public health problem, making weight loss a common apprehension today, covering almost all age groups. In view of this, the idea of rapid weight loss arises, impacting on the emergence of popular diets to eradicate obesity. Objective of this work was to demonstrate through several studies the real reasons for the unrestrained practice of restrictive diets and their negative repercussion in the development of eating disorders. Review: A review of the literature was carried out with the aim of approaching the thematic fad diets and their effects on the health of their followers. Discussion: The yearning for weight loss embedded in societies is perceived by the literature as one of the factors that would contribute to the disordered growth of these disorders nowadays. Final considerations: It is noted that regardless of the motivation to practice restrictive diets, they acquire a connotation of tolerance, making a satisfactory quality of life impossible.
\end{abstract}

Keywords: restrictive diets, fad diets, healthy eating index, analysis of fad diets, eating disorders.

\section{Introdução}

O sobrepeso e a obesidade são um problema atual de saúde pública nas várias populações, tornando a perda de peso uma apreensão comum abrangendo quase todas as faixas etárias. Diante deste panorama, surge a ideia de perda ponderal rápida sem a adesão de estratégia de redução da ingestão de energia derivada da dieta e acréscimo do gasto energético a partir da prática regular de atividade física (Viana et al., 2013). Impactando diretamente no surgimento de dietas populares para erradicar a obesidade ao longo das últimas três décadas (Ovbiosa-Akinbosoye \& Long, 2011).

As dietas populares podem ser reunidas de acordo com a proporção dos macronutrientes preconizada (OvbiosaAkinbosoye \& Long, 2011). Conquanto muitas das dietas para emagrecimento sejam eficientes na redução em curto prazo, a estimativa qualitativa dessas dietas muitas vezes não é demonstrada ou sabida. De fato, o exame mais detalhado da composição nutricional dos planos alimentares sugeridos pelas dietas populares despontou que nenhum deles atinge um apropriado índice de alimentação saudável (Previato et al., 2014).

O Índice de alimentação saudável (IAS) é uma medida abreviada da qualidade da dieta e pode ser usado para qualificar e conduzir a ingestão da dieta individual e populacional e, dessa forma, incentivar a saúde e também evitar doenças, sejam elas crônicas não transmissíveis (DCNT) ou não, por meio de programas de educação nutricional (Viana, 2014). 
Inúmeros indivíduos buscam uma dieta de emagrecimento porque anseiam desesperadamente por uma aparência mais atraente e "sociável". A imagem corporal deve ser fruto de uma vida saudável e uma alimentação equilibrada, não devendo de formal alguma, ser encarada como resultado de dietas milagrosas, remédios ou prática extenuante e autoflagelada de exercícios físicos (Pereira, 2016).

Essas dietas são práticas alimentares populares e temporárias que prometem resultados rápidos e desejados, contudo necessitam de conhecimento científico (Escott-Stump, 2011).

Há uma variedade de dietas da moda citadas em revistas e blogs, todas cultuando e enaltecendo o emagrecimento rápido e indolor, sabe-se que a adesão a estas é grande no começo, devido à maior parte ser extremamente restritiva e agressiva, contudo essas tentativas tornam-se frustradas, pois não levam em consideração a rotina dos adeptos e tampouco seus hábitos alimentares (Santos, 2010).

Outros enfoques importantes são aos sinais clínicos que as dietas com redução drástica de energia e nutriente, seja a médio e longo prazo, apresentam. Como desequilíbrios metabólicos, dentre eles podemos citar a formação de corpos cetônicos e sobrecarga hepática e renal (Santos, 2010).

Não existe comprovação cientifica indicando que dietas ricas em gorduras e proteínas e pobres em carboidrato tenham uma vantagem metabólica em relação as dietas convencionais para a perda de peso, podendo inclusive contribuir para o aparecimento de doenças crônicas entre elas obesidade, diabetes, colesterol e doenças cardiovasculares (Santos, 2010).

Observa-se que o estimulo inicial para a mudança da alimentação e a redução de peso pode ser de caráter social (cobrança excessiva da família, namorado e amigos) mas, também pode partir de educadores físicos, professores de balé, treinadores de luta, etc. Assim, devido a uma insatisfação corporal e/ou uma "necessidade profissional”, iniciam-se as dietas restritivas. Subentende-se então que a dieta surge única e exclusivamente para favorecer a perda de peso (Santos, 2010).

Nos dias atuais as diversas variedades de dietas populares surgem do desespero coletivo e acompanhando esse crescimento estão os transtornos alimentares, ambos representando insegurança e insatisfação quanto ao corpo (Carvalho et al., 2009).

Os transtornos alimentares são fenômenos multidimensionais decorrentes da interação de fatores pessoais, familiares e socioculturais, caracterizados pela apreensão intensa com alimento, peso e corpo. Os tipos de transtornos alimentares são: anorexia nervosa (AN), bulimia nervosa (BN) e transtornos alimentares não especificados, que incluem o transtorno do comer compulsivo (Almeida, 2015).

Atrelada ao padrão do corpo magro, são imputadas as mensagens de sucesso, controle, aceitação, conquistas de amor e estabilidade psicológica. Assim, o indivíduo espera que, com a magreza, alcançará todas essas qualidades positivas, sendo a prática de dietas recomendada como solução para todas as dificuldades. E, no malogro desse intuito, tal indivíduo é visto como inábil e sem autocontrole (Manuel, 2016).

Entretanto, o modelo imposto como perfeito não respeita as diferentes compleições e induz as pessoas a se sentirem gordas e almejarem o emagrecimento. Portanto, a insatisfação corporal leva muitas pessoas a caírem no artifício das dietas, sendo esse desprezo quanto aos seus corpos apoiado pela indústria do emagrecimento, que difunde os mitos acerca das dietas (Baptista, 2013). Diante deste contexto, este trabalho tem por objetivo relacionar a prática de dietas restritivas ao surgimento e agravamento de transtornos alimentares, seja a anorexia, a bulimia ou o transtorno de comer compulsivo.

\section{Revisão}

Foi realizada uma revisão da literatura de forma exaustiva e sistemática, com o intuito de abordar a temática dietas da moda e os seus efeitos deletérios sobre a saúde dos seus seguidores, fazendo uso das palavras chaves: dieta da moda, dieta restritiva, transtornos alimentares, tipos de dietas, fatores que levam aos distúrbios alimentares, nos idiomas português, inglês e espanhol. As informações foram obtidas somente em fontes primárias indexadas nas bases de dados SciELO, PubMed, Medline, Lilacs, Dedalus e ISI Web of Knowledge tais documentos referem-se aos últimos dez anos, a busca gerou um total de 56 artigos tendo início no mês de janeiro e sendo finalizada no mês de agosto.

Após a leitura exploratória de todos os resumos, alguns inicialmente selecionados não foram incluídos devido à dificuldade de acesso, por não estarem disponíveis em sua íntegra nas bases pesquisadas ou não conterem conteúdo relevante para o presente trabalho. Foram selecionados apenas aqueles que contemplassem mais diretamente a temática e que estivessem disponíveis nas bases citadas, totalizando assim 33 artigos. 


\section{Discussão}

\section{Dietas restritivas nas patologias}

Vários estudos abordam as doenças crônicas e a interligação desta com as restrições dietéticas impostas pela doença. Neste quesito, o abandono pela família e sociedade ou o excesso de zelo, podem prejudicar o tratamento e a reabilitação do convalescente (Vieira et al., 2011).

Uma grande parcela desta população se julgam vítimas da condição imposta pela mazela adquirida, comparam-se então a outras pessoas, e chegam à conclusão de que suas dietas e práticas alimentares sejam análogas ou melhores, julgando-se submetidos à sua condição de portadores de injúrias incuráveis, especialmente nos casos de obesidade (Vieira et al., 2011).

Existem sim indicações de dietas restritivas, mas, estas tendo um cunho preponderante no tratamento e melhora de algumas patologias, dentre elas, destacam-se: a doença celíaca, intolerância a lactose e a epilepsia.

O tratamento da DC consiste na absoluta e terminante exclusão do glúten da dieta. A eliminação do glúten da dieta deve ser realizada tanto por pacientes sintomáticos quanto por assintomáticos, para garantir uma boa qualidade de vida e minimizar os riscos futuros de morbidade e mortalidade (Andreoli et al., 2013).

A dieta isenta de glúten deve atingir as necessidades nutricionais do paciente celíaco, assegurando nutrição adequada por atender as necessidades de macro e micronutrientes (Andreoli et al., 2013).

Obviamente, a adesão à dieta isenta de glúten exige uma ampla mudança no hábito alimentar dos pacientes celíacos. Estudos mostraram que a adesão dietética por parte destes, nem sempre é seguida à risca, ocorrendo transgressões frequentes, sobretudo na adolescência (Andreoli et al., 2013).

$\mathrm{O}$ aparecimento de sintomas gastrointestinais pela intolerância a lactose qualifica a intolerância à lactose (Mattar \& Mazo, 2010).

Nestes casos recomendam-se então evitar leite e produtos lácteos da dieta para se obter melhora dos sintomas. Devido à presença de alimentos com lactose não ser detectada na sua composição, o tratamento pode ter sua acurácia diminuída e/ou interrompida (Mattar \& Mazo, 2010).

Ao longo dos anos vários autores enfatizam que pacientes epilépticos apresentam um progresso em suas crises durante o jejum ou mesmo pela acidose metabólica levada pelo jejum. Foi proposta então uma dieta que representasse as alterações bioquímicas especificas dos períodos de jejum, sendo está a dieta cetogênica (Rola \& Vasconcelos, 2014).

Sua indicação é voltada especialmente para crianças com diversas crises epilépticas de difícil controle, que não mostram melhora junto às medicações habituais. Pode, ainda, ser indicada para pacientes que se demonstrem intolerantes aos efeitos crônicos das drogas antiepilépticas (Rola \& Vasconcelos, 2014).

Aspectos negativos da dieta são observados em pacientes que obrigatoriamente dependam desta restrição para efetiva melhora e remissão da doença e seus sintomas, tornando-se então um agente bloqueador de um modelo de vida saudável. A dieta agregada a emoções de raiva e tristeza, perante as restrições alimentares atribuídas pela doença, foi apontada por alguns pacientes como danosa à saúde. Outro fator contraproducente são os inúmeros relatos equivocados sobre uma alimentação saudável ser muito onerosa e que tal seja privilégio para pessoas ricas (Vieira et al., 2011).

Os alimentos doces foram identificados como os mais atraentes e desejados, ao transpassarem o sentido de impedimento e restrição de uma fonte de prazer em seu cotidiano, transfigurando-se em algo ainda mais atrativo, justificando a difícil e custosa adesão ao tratamento, se tornando o maior adversário da prescrição dietética (Vieira et al., 2011).

Em alguns estudos revisados, a exclusão de determinados alimentos da prática alimentar representa, para alguns grupos populacionais, um benefício à saúde, mas admitem que o cumprimento dessa restrição é difícil no dia-a-dia, justificando esta dificuldade pela sua implicação na liberdade e no prazer da comensalidade (Vieira et al., 2011).

\section{Dietas restritivas na busca pelo corpo perfeito}

Um padrão de alimentação balanceada deve satisfazer alguns conceitos basilares para que não acarretem deficiências nutricionais e, por conseguinte, agravos à saúde. Ponderando sobre tal alicerce alimentar, 
estabeleceu-se quatro leis que regem uma boa alimentação, sendo elas: lei da quantidade, lei da qualidade, da harmonia e da adequação (Lima, 2009).

Estudiosos afirmam que dietas indistintas, sem controle de macro e micronutrientes e com valor energético diminuído acutilam as leis de quantidade e qualidade, não alcançam requerimentos nutricionais característicos das faixas etárias e não propiciam hábitos de vida saudáveis (Lima, 2009).

As dietas com restrição calórica exacerbada podem acarretar sintomas, como: queda no cabelo, fadiga, constipação, diarreia, pele seca, anemia e irregularidade no ciclo menstrual. Tais sintomas estão associados à oferta insuficiente de micronutrientes (Betoni et al., 2010).

De acordo com análises de cardápios publicados em revistas não científicas, a maior parte deles eram hiperproteicos, e apenas uma pequena porcentagem encontrava-se adequado. Fato preocupante, já que o consumo elevado de proteínas eleva a concentração do mineral cálcio na urina e também pode estar correlacionado a quadros de aterosclerose, doenças renais, câncer e osteoporose (Marangoni \& Maniglia, 2017).

$\mathrm{O}$ termo restrained eating foi delineado como uma tendência a reduzir o consumo alimentar conscientemente, precavendo-se de aumentar o peso corpóreo ou estimular sua perda (Moore, 2014).

Estudos evidenciaram que unrestrained eaters, pessoas que não reduzem o consumo de alimentos, ou nondieting, pessoas não adeptas a dietas, ingerem uma menor quantidade após um lanche hipercalórico. Esta coordenação energética é natural, isto é, a pessoa contrabalança o lanche comendo menos, posteriormente. Entretanto, restrained eaters, pessoas que diminuem a ingesta alimentar, se alimentam mais depois de um lanche hipercalórico. Tal comportamento é esclarecido pela expansão do controle de cognição, já que pressupõem ter excedido o consumo alimentar recomendado com a ingestão da refeição energética. Tal circunstância pode originar hora uma cadeia de compulsão alimentar, hora restrição energética (Moore, 2014).

\section{Índice de alimentação saudável}

O índice de alimentação saudável foi elaborado por Kennedy (2008), objetivando construir um índice de qualidade geral da dieta que englobasse, em uma única medida, as necessidades nutricionais e os guias dietéticos para consumidores norte-americanos (Kennedy, 2008).

É considerado um instrumento de medida simples e concisa da qualidade da dieta, que pode ser utilizado para verificar as mudanças nos padrões de consumo e também é reconhecido como instrumento útil na educação nutricional e na promoção a saúde. As informações dietéticas são analisadas em um recordatório de 24 horas e em um registro de dois dias de alimentos (Kennedy, 2008).

O IAS é constituído por 10 elementos, que se baseiam em distintos aspectos de uma alimentação saudável. Os componentes de 1 a 5 avaliam a dieta de acordo com as recomendações de porções da pirâmide americana para os 5 principais grupos: cereais, hortaliças, frutas, leite e carnes. Os componentes 6 e 7 medem, respectivamente, a porcentagem calórica proveniente de gordura total e de gordura saturada. Os componentes 8 e 9 baseiam-se na ingestão de colesterol e de sódio em miligramas. O componente 10 avalia a diversidade da dieta, que se refere ao número de itens diferenciados consumidos em um período de três dias (Kennedy, 2008).

Logo, o escore máximo é obtido quando houver o consumo de 6-11 porções de cereais; 3-5 porções de vegetais; 2-4 porções de frutas; 2-3 porções de leite e derivados; e 2-3 porções de carne. Todavia, o escore de zero é obtido quando o consumo for nulo, ou seja, quando não houver consumo desses itens. Para a pontuação dos componentes 6 a 10, o indivíduo recebe um escore máximo de 10 quando o consumo de gordura total for menor ou igual a $30 \%$ do valor calórico total (VCT); gordura saturada for menor que $10 \%$ do VCT; colesterol for menor que $300 \mathrm{mg}$; sódio menor que $2400 \mathrm{mg}$; e a variedade da dieta for igual ao consumo de 16 itens diferentes no período de três dias. Por outro lado, o indivíduo recebe o escore zero quando o consumo de gordura total for maior ou igual a $45 \%$ do VCT; gordura saturada for maior ou igual a $15 \%$ do VCT; colesterol for maior ou igual a $450 \mathrm{mg}$; sódio maior ou igual a $4800 \mathrm{mg}$; e a variedade da dieta for menor ou igual a 6 itens (Kennedy, 2008).

Para cada elemento há uma pontuação, que varia de zero a 10 e, dessa forma, o índice varia de zero a 100. Quando o IAS for 80, o escore é considerado satisfatório. Escores que variam entre 51 e 80 demonstram a necessidade de melhorar a qualidade da dieta, enquanto os menores que 51 indicam qualidade da dieta insatisfatória (Kennedy, 2008).

Dentre as vantagens do IAS tem-se que o método se embasa nas mais recentes recomendações dietéticas publicadas. Portanto, é capaz de obter informações para qualificar a dieta como excelente ou péssima, de 
maneira sucinta e uniforme. O IAS ainda possibilita avaliar a qualidade total da dieta de indivíduos e não exclusivamente de componentes isolados (Kennedy, 2008).

\section{Análise do uso das dietas da moda através do índice de alimentação saudável}

Em um estudo prospectivo sobre a adequação nutricional de dietas publicadas por revistas não científicas realizado por Silva et al., 2012 quanto aos macronutrientes, averiguou-se que a maioria $(91,7 \%)$ das dietas se apresentaram hipoglicídicas, sendo que as demais $(8,3 \%)$ se encontraram normoglicídicas. No caso da proteína, $(91,7 \%)$ de dietas eram hiperproteicas e $(8,3 \%)$ normoproteicas. Em relação aos lipídios, observouse que $(91,7 \%$,) das dietas eram hiperlipídicas e $(8,3 \%)$ mostravam-se hipolipídicas. No quesito classificação das dietas, segundo os critérios do IAS, verificou-se que a maior parcela $(66,7 \%)$ encontrava-se carecendo de alterações e apenas 33,3\% estavam inadequadas (Silva \& Kirsten, 2016).

Com relação aos micronutrientes, constataram um consumo médio inadequado de cálcio (mg) e ferro (mg) (Silva \& Kirsten, 2016).

No quesito sazonalidade, a distribuição de cálcio mostrou-se expressivamente elevada nas dietas de inverno. Quanto aos macronutrientes, constatou-se que as dietas publicadas no verão se caracterizavam em: hipoglicídicas $(12,9 \%)$, hiperprotéicas $(7,7 \%)$ e hiperlipídicas $(14,3 \%)$; em relação às calorias, as dietas publicadas no verão exibiram valores calóricos inferiores (1278,97 Calorias) ao achado nas dietas publicadas no inverno (1401,73 Calorias) (Silva \& Kirsten, 2016).

Comumente essas restrições promovem uma rápida redução de peso, isso se deve a ingesta calórica reduzida, principalmente por parte dos carboidratos, o organismo poupa então as proteínas, importantes na manutenção e construção tecidual e imunológica, mobilizando a gordura e usando-a como fonte primária de energia (Escott-Stump, 2011).

Essa drástica redução calórica acaba produzindo em excesso as cetonas urinárias, que se envolvem na liberação renal do ácido úrico, elevando os níveis séricos desse ácido, podendo acarretar o surgimento de hiperuricemia. Atrelado a isso, outras reações podem ser notadas em dietas de valor calórico muito baixo, dentre eles podemos mencionar: a diminuição do débito cardíaco, frequência cardíaca e pressão arterial; diminuição do potássio corporal total; intolerância ao frio; queda de cabelo; fadiga; dificuldade de concentração; nervosismo; constipação ou diarreia; pele seca; unhas fracas; flacidez e tontura (Escott-Stump, 2011).

Mais uma vez, não existe evidência científica indicando que dietas hiperprotéicas, hiperlipídicas e hipoglicídicas, encontradas no presente estudo, possuam um benefício metabólico sobre as dietas estudadas e recomendadas para redução de peso. Tal perda se deve a diminuição calórica, em grande parte proveniente dos carboidratos (Escott-Stump, 2011).

Observa-se então que a maioria das dietas restritivas, apresentam severa ausência dos grupos alimentares essenciais, culminando em déficits nutricionais entre eles ferro, vitamina A e zinco. Trazendo diversos agravos à saúde dos indivíduos, tais como o aparecimento de doenças crônicas não transmissíveis, entre elas diabetes, obesidade, câncer, acidente vascular cerebral (AVC), estando conexas aos motivos mais recorrentes de morte registradas atualmente (Viana, 2014).

Os achados de Marangoni \& Maniglia (2017), corroboram com os dados supracitados, já que seus resultados apresentaram níveis insuficientes dos minerais ferro e cálcio em todos os cardápios avaliados na pesquisa, em relação ao zinco, das 24 publicações, somente uma revista alcançou a recomendação, quanto aos macronutrientes, uma apresentou o menor valor de lipídio e três mostraram-se hiperlipídicas. As maiores discrepâncias foram com relação ao carboidrato e ao valor energético, a grande maioria apresentando um valor abaixo do recomendado para a população em geral (Marangoni \& Maniglia, 2017).

As dietas indicadas pelo "Dr. Ornish", "Vigilantes do Peso ${ }^{\circledR}$ " e a "Revolução da ${ }^{\circledR}$ Glicose" aparentemente apresentam ter os melhores índices de alimentação saudável (Almeida et al., 2009) (Tabela 1).

\section{Fatores predisponentes para o surgimento dos transtornos alimentares}

Sociólogos têm sugerido dois processos que originam atitudes e comportamentos: reforço social e a modelagem. O primeiro refere-se ao meio pelo qual as pessoas interiorizam atitudes e comportam-se por meio da aprovação daqueles a sua volta (Almeida et al., 2013). 
Tabela 1. Composição nutricional e índice de alimentação saudável da dieta dos planos alimentares propostos pelas principais dietas populares de emagrecimento.

\begin{tabular}{|c|c|c|c|c|c|c|c|c|c|}
\hline \multirow[b]{2}{*}{ Características das dietas } & \multirow[b]{2}{*}{$\begin{array}{l}\text { Revolução da } \\
\text { glicose }\end{array}$} & \multicolumn{2}{|c|}{ Vigilantes do peso } & \multicolumn{2}{|c|}{ Atkins } & \multicolumn{2}{|c|}{ South Beach } & \multirow[b]{2}{*}{ Ponto Z } & \multirow[b]{2}{*}{ Ornish } \\
\hline & & Rica em carboidratos & $\begin{array}{l}\text { Rica em } \\
\text { proteínas }\end{array}$ & $\begin{array}{c}45 \mathrm{gde} \\
\text { carboidratos }\end{array}$ & $\begin{array}{c}100 \mathrm{gde} \\
\text { carboidratos }\end{array}$ & c & Fase 3 & & \\
\hline IAS ajustado para energia & $54,6 \pm 3,4$ & $60,4 \pm 3,5$ & $51,2 \pm 3,7$ & $41,6 \pm 3,2$ & $42,1 \pm 3,7$ & $53,0 \pm 3,3$ & $46,4 \pm 3,2$ & $54,9 \pm 3,9$ & $61,9 \pm 3,4$ \\
\hline \multicolumn{10}{|l|}{ Grupos alimentares (porções/dia) } \\
\hline Vegetais & $6,0 \pm 1,9$ & $6,3 \pm 1,2$ & $3,1 \pm 0,7$ & $6,6 \pm 1,1$ & $6,4 \pm 2,6$ & $11,1 \pm 5,8$ & $6,4 \pm 3,0$ & $7,2 \pm 2,0$ & $12,6 \pm 3,2$ \\
\hline Frutas & $5,1 \pm 1,4$ & $3,1 \pm 2,8$ & $2,4 \pm 2,3$ & $1,9 \pm 0,3$ & $3,0 \pm 0,4$ & $2,8 \pm 0,9$ & $2,5 \pm 1,2$ & $4,2 \pm 2,1$ & $4,7 \pm 2,0$ \\
\hline Nozes e proteína de soja & $4,1 \pm 1,7$ & $1,4 \pm 1,1$ & $1,3 \pm 1,2$ & $2,2 \pm 1,9$ & $3,1 \pm 2,4$ & $1,3 \pm 1,5$ & $0,4 \pm 0,4$ & $2,0 \pm 1,8$ & $2,1 \pm 1,2$ \\
\hline Carnes branca evermelha & $1,5 \pm 1,9$ & $3,4 \pm 1,5$ & $2,3 \pm 2,1$ & $1,0 \pm 0,8$ & $1,2 \pm 1,4$ & $2,6 \pm 1,7$ & $2,5 \pm 2,2$ & $1,8 \pm 1,9$ & $4,0 \pm 0,0$ \\
\hline \multicolumn{10}{|l|}{ Composição nutricional } \\
\hline Total de energia (kcal/dia) & $1729 \pm 133$ & $1204 \pm 104$ & $1118 \pm 79$ & $1547 \pm 218$ & $1858 \pm 253$ & $1271 \pm 263$ & $1404 \pm 273$ & $1025 \pm 122$ & $1739 \pm 144$ \\
\hline Carboidratos (\% energia) & $57,1 \pm 3,1$ & $59,0 \pm 4,6$ & $52,9 \pm 4,1$ & $22,2 \pm 3,4$ & $29,5 \pm 5,1$ & $41,4 \pm 8,7$ & $37,0 \pm 10,2$ & $43,6 \pm 4,7$ & $81,0 \pm 2,3$ \\
\hline Proteínas (\% energia) & $19,5 \pm 1,4$ & $21,0 \pm 2,8$ & $25,8 \pm 4,5$ & $25,3 \pm 3,4$ & $20,7 \pm 3,1$ & $24,2 \pm 5,7$ & $25,7 \pm 5,3$ & $30,8 \pm 2,6$ & $15,9 \pm 1,6$ \\
\hline Lipídeos (\% energia) & $28,8 \pm 2,8$ & $25,7 \pm 2,2$ & $24,8 \pm 3,3$ & $54,9 \pm 5,7$ & $52,6 \pm 6,6$ & $37,4 \pm 8,8$ & $39,6 \pm 8,6$ & $29,2 \pm 3,9$ & $6,5 \pm 1,8$ \\
\hline Saturados (\% energia) & $5,3 \pm 1,0$ & $5,6 \pm 2,5$ & $6,0 \pm 2,6$ & $16,2 \pm 2,2$ & $17,6 \pm 3,5$ & $9,6 \pm 2,6$ & $10,4 \pm 3,1$ & $7,6 \pm 1,6$ & $1,5 \pm 0,6$ \\
\hline Trans (\% energia) & $0,4 \pm 0,4$ & $0,3 \pm 0,3$ & $0,3 \pm-0,3$ & $1,2 \pm 0,7$ & $1,3 \pm 0,4$ & $0,5 \pm 0,3$ & $0,6 \pm 0,3$ & $0,3 \pm 0,2$ & $0,3 \pm 0,2$ \\
\hline Monoinsaturados (\% energia) & $13,7 \pm 1,9$ & $11,2 \pm 1,7$ & $11,6 \pm 1,2$ & $21,9 \pm 4,3$ & $20,8 \pm 4,7$ & $16,0 \pm 4,3$ & $18,4 \pm 5,8$ & $13,5 \pm 1,5$ & $1,8 \pm 0,8$ \\
\hline Poli-insaturados n3 (\% energia) & $1,8 \pm 2,0$ & $1,2 \pm 0,7$ & $1,0 \pm 0,9$ & $3,4 \pm 0,7$ & $3,4 \pm 0,7$ & $2,7 \pm 2,3$ & $1,8 \pm 0,7$ & $1,3 \pm 0,9$ & $0,6 \pm 0,1$ \\
\hline Poli-insaturados/saturados (\% energia) & $1,6 \pm 0,7$ & $1,3 \pm 0,6$ & $1,0 \pm 0,4$ & $0,8 \pm 0,1$ & $0,6 \pm 0,2$ & $1,0 \pm 0,4$ & $0,9 \pm 0,4$ & $0,7 \pm 0,2$ & $0,7 \pm 0,2$ \\
\hline Fibras totais (g/dia) & $39,8 \pm 8,0$ & $34,7 \pm 9,3$ & $22,9 \pm 6,8$ & $22,1 \pm 4,4$ & $27,6 \pm 4,2$ & $26,0 \pm 9,0$ & $20,4 \pm 4,4$ & $21,8 \pm 3,2$ & $48,4 \pm 6,4$ \\
\hline Fibras de cereais (g/dia) & $8,3 \pm 7,6$ & $12,4 \pm 8,4$ & $8,5 \pm 5,9$ & $1,8 \pm 1,5$ & $6,9 \pm 3,4$ & $4,9 \pm 4,1$ & $6,4 \pm 5,4$ & $2,0 \pm 5,2$ & $11,3 \pm 9,0$ \\
\hline Sódio (mg/dia) & $1749 \pm 541$ & $1653 \pm 356$ & $1790 \pm 491$ & $2251 \pm 545$ & $2539 \pm 830$ & $1669 \pm 789$ & $1815 \pm 676$ & $1726 \pm 738$ & $2206 \pm 723$ \\
\hline
\end{tabular}

Fonte: Almeida et al. (2009).

Um ótimo exemplo disso é a lavagem cerebral que a mídia faz, enaltecendo a magreza, conjecturando-a a sucesso, felicidade e determinação, mostrando ao jovem o que ele tem que ser e qual caminho trilhar para chegar lá, e assim que alcançar o almejado peso, o indivíduo será aceito e amado.

A modelagem remete-se ao processo em que o indivíduo analisa comportamentos alheios e os imita. A sociedade passa então a ser um exemplo de apreensões excessivas com as medidas corporais, através de dietas exageradas, comportamentos prejudiciais de preocupação extrema com o ganho de peso e compulsões alimentares (Almeida et al., 2013).

Concernente as diversas causas que podem levar ao surgimento dos transtornos alimentares, foi comprovado que uma pequena porcentagem está ligada a fatores genéticos, porém é desconhecido o tamanho de sua contribuição para a incursão da doença. Porém sabe-se que estes transtornos, na maioria das vezes, são uma sequela multifatorial, em especial, dos fatores socioculturais e psíquicos (Oliveira \& Hutz, 2010).

Os transtornos alimentares advêm de comportamentos representados por aspectos como medo excessivo do ganho de peso e a forma corporal, diminuição voluntária do consumo energético com avançada perda de peso, ingesta descontrolada de alimentos seguida de vômitos e uso indiscriminado de laxantes e/ou diuréticos (Oliveira \& Hutz, 2010).

São doenças graves e de prognóstico insatisfatório, que promovem altos índices de letalidade e suscitam limitações emocionais, sociais e físicas (Oliveira \& Hutz, 2010).

$\mathrm{O}$ anseio pelo emagrecimento incrustado nas sociedades, é percebido pela literatura como um dos fatores culturais centrais que colaborariam para o crescimento desordenado destes transtornos na atualidade (Oliveira \& Hutz, 2010).

O estudo das alterações dos padrões estéticos evidência, a partir dos anos 60, uma idealização de imagem feminina esquálida, consolidada nos manequins e modelos, que vêm assumindo compleições físicas cada vez menores (Oliveira \& Hutz, 2010).

Á partir da década de 80, o aumento da busca pela magreza já era perceptível. Em pesquisas que colacionavam o peso e as formas corporais de candidatas do concurso "Miss América" e modelos da capa de uma revista masculina do ano de 1959 ao ano de 1978, verificaram uma modificação progressiva no padrão estético. Os autores do estudo verificaram uma mudança paulatina das formas corporais curvilíneas para um padrão corporal cada vez mais esguio e sem formas definidas (Oliveira \& Hutz, 2010).

O final do século XX e início do século XXI são caracterizados pela veneração ao corpo. Existe então uma procura pelo corpo perfeito alcançada de maneira compulsiva, transformando-se em uma rotina para muitas pessoas, particularmente o público feminino, pertencentes a classes médias urbanas. O preconceito contra a obesidade se torna palpável, e a magreza é tida como uma imagem de sucesso, perfeição competência, autocontrole e atratividade sexual (Oliveira \& Hutz, 2010).

Esse arquétipo inatingível de magreza, vastamente disseminado pela mídia, já se perpetuou no inconsciente coletivo e confinou as pessoas dentro de si mesmas. Em razão disso, mais de $98 \%$ das mulheres não se veem bonitas, sendo expostas e bombardeadas por ideais estéticos característicos de um público diminuto, tornando-as um fantoche da tecnologia da produção de massa (Oliveira \& Hutz, 2010). 
Existe ainda uma analogia entre a mídia e a urbanização na propensão aos transtornos alimentares. Deveras, um maior grau de urbanização parece potencializar a probabilidade dessas patologias, levando a uma substancial exposição ao ideal de beleza através da mídia, implicando em modificações de hábitos alimentares, sedentarismo e um excessivo número de pessoas com obesidade (Oliveira \& Hutz, 2010).

Essa procura compulsiva sobrepuja, assim, o delgado limite entre um zelo saudável com o corpo e a perspicaz cinesia da instalação dos transtornos alimentares. Tal modelo estético é tido pela literatura como principal na expansão do número de casos de tais transtornos, sendo os mais citados: a anorexia nervosa, bulimia nervosa e a compulsão alimentar (Oliveira \& Hutz, 2010).

A Anorexia Nervosa é um transtorno definido por uma alimentação limitada onde o principal enfoque dos indivíduos anoréxicos é sustentar um peso corporal baixo em função do temor em engordar, onde a modificação da imagem corporal colabora para que eles continuamente se enxerguem muito acima do peso. Comumente esse transtorno se desenvolve na adolescência a partir da puberdade e também em adultos jovens (Damasceno, 2010).

Na AN determinadas variações endócrinas, como, diminuição de alguns hormônios do eixo hipotálamohipófise gonadal, são suscetíveis de complicações clínicas tendo a capacidade de acarretar amenorreia, que pode ser seguida por inúmeros retrocessos para fases pré-puberais, como redução do ovário, do volume das mamas, do útero, atrofia da parede vaginal, queda dos pelos pubianos e até infertilidade com possíveis ovulações. Possui consequências cardiovasculares como bradicardia, resposta diminuída ao exercício físico, atrofia do músculo cardíaco entre outros. Alterações gastrointestinais, dilatação e esvaziamento gástrico lento, lactase e lipase diminuídas, entre outras. Problemas hematológicos como anemia e leucopenia; metabólicos como hipercolesterolemia, hipoglicemia e hipercarotenemia; hidroeletrolíticos, dentre eles desidratação e hipotermia; comprometimentos cerebrais como, atrofia; problemas renais e até morte súbita (Damasceno, 2010).

Na terapêutica a nível ambulatorial, os propósitos da reabilitação nutricional incluem: restabelecimento da compleição física, supressão dos comportamentos para perda de peso, evolução nos comportamentos alimentares e progresso no estado psicológico e emocional. Somente a recuperação do peso não implica em recuperação total e compelir o paciente a simplesmente recobrá-lo sem suporte psicológico não é recomendável (Bechara \& Kohatsu, 2014).

A bulimia nervosa constitui-se num TA cujo indivíduo exibe episódios de compulsão alimentar, consumindo alimentos em quantidades atípicas, acompanhado de condutas purgativas como vômitos auto induzidos e uso indiscriminado de laxantes ou diuréticos, restrição alimentar ou ainda períodos de jejum. Paralelamente a essas características está o medo de engordar e a apreensão com a imagem corporal. Tal comportamento nocivo pode advir de incessantes tentativas de dietas de emagrecimentos (Damasceno, 2010).

As mudanças físicas mais frequentes exibidas por indivíduos com esta patologia são os tilomas evidentes no dorso da mão, conhecida como "sinal de Russell”, por suscitar o reflexo do vômito; expansão das glândulas parótidas e a corrosão no esmalte dentário, ocasionado pelos ácidos gástricos. Ademais, apresentam queda de cabelo, alterações menstruais, descamação na pele, edemas, entre outros (Damasceno, 2010).

Concernente às consequências médicas da $\mathrm{BN}$, as mais habituais são as alterações metabólicas e hidroeletrolíticas, dentre elas baixa concentração de sódio, magnésio, cloro e potássio no sangue podendo originar falência cardíaca e outras alterações. O vômito excessivo pode induzir perfurações no esôfago, estômago e pneumomediastino, bem como a dilatação do mesmo pode promover alterações de outros órgãos como rins e fígado, e também anormalidades intestinais em função do excesso de laxativos. A má-nutrição também alcança alguns indivíduos com esse transtorno, podendo gerar alterações hematológicas como anemia e problemas imunológicos, em relação a prática extenuante de exercícios, estes podem lesar músculos e articulações. Por último vem as alterações visuais e diminuição da atividade do sistema nervoso simpático (Damasceno, 2010).

A educação nutricional permeia a terapia cognitiva indicada para o tratamento da BN. Ela oportuniza a orientação alimentar para a implantação de padrões nutricionais adequados. Seus principais propósitos são: a erradicação do ciclo "compulsão alimentar - purgação", a fixação de um padrão alimentar apropriado e as mudanças atitudinais relacionadas a alimentação e espaço corporal. A longo prazo, intenta-se fazer com que a ingesta volte (ou venha a ser) um ato natural, não obstante a mídia que apregoa a normalidade das dietas restritivas (Bechara \& Kohatsu, 2014).

A compulsão alimentar é um TANE que se caracteriza pela ingestão de uma quantidade exorbitante de comida, em contraste com a quantidade que outras pessoas ingeririam em um curto espaço de tempo, ao menos duas vezes por semana, por seis meses consecutivos, seguido do sentimento de ausência de autocontrole. Nesse ínterim, os indivíduos experimentam uma sensação de descontrole sobre o quê ou o quanto comem e, ao final, sentem-se culpados e depressivos (Cortez et al., 2011). 
Contrariamente à BN, na compulsão alimentar o indivíduo não utiliza métodos para contrabalançar a quantidade excessiva de alimentos ingeridos (Cortez et al., 2011).

É crucial explanar que a família e os amigos podem tanto propiciar fatores de proteção contra o incremento de distúrbios alimentares, quanto imputar o seu estabelecimento, dependendo de como é o relacionamento que têm com o indivíduo propenso a apresentar o TA (Leonidas \& Santos, 2015).

Neste contexto, debate-se então a concepção do apoio social, referente ao indivíduo se sentir amado, cuidado, valorizado e estimado pela sociedade que o rodeia. Sendo encarado como um considerável fator protetor contra distúrbios emocionais e alimentares (Leonidas \& Santos, 2015).

Dentre os vários grupos de risco para o desenvolvimento de TA, podemos citar: os estudantes de nutrição, esportistas, bailarinos, crianças e adolescentes. Possivelmente as graduandas de Nutrição pertençam a este grupo por apresentarem, na maioria das vezes, uma preocupação excessiva com a sua configuração física, estando em contínuo contato com os alimentos, perpetuando a neurose por uma aparência engendrada pela sociedade, como se a compleição física do Nutricionista definisse sua experiência e êxito no ramo. As futuras nutricionistas têm familiaridade com os nutrientes presentes nos alimentos, cobrando-se incessantemente para que esse conhecimento teórico vire o quanto antes uma prática produtiva. Optando por se enveredarem em caminhos alternativos para alcançarem o que acreditam ser os seus objetivos pessoais e profissionais, na maioria das vezes colocando sua saúde em risco. Logo a conjuntura que essas alunas estão vivenciando, desde a entrada na faculdade até a vivência da vida profissional, contribui com o desenvolvimento de distúrbios nutricionais (Silva et al., 2012).

Bosi et al. (2014), compararam o comportamento alimentar de estudantes da área da saúde com os da área de humanas, e o estudo revelou que os que cursavam Nutrição, Medicina ou outras áreas contíguas, demonstraram maior probabilidade de desenvolver transtornos do que os que cursavam Administração, Letras, etc. Tal fato se daria pela procura dos cursos de Nutrição por pessoas que possam ter maior propensão a desenvolver esses transtornos. A investigação apontou para uma maior existência de distorção da imagem corporal e de comportamento alimentar inadequado entre as alunas de nutrição (Bosi et al., 2014).

A prática de atividade física exaustiva também é regularmente usada nas práticas de TA, sendo o treinamento físico sistemático seguido à risca por esportistas e também um potencializador de alterações morfológicas e estas, por conseguinte, serem adotadas como alternativas empregadas para atenuar o desagrado com a aparência corporal, podemos considerá-los então como um grupo de risco para desencadeamento de TAs como a anorexia nervosa (AN) e a bulimia nervosa (BN) (Fortes et al., 2014).

A predisposição do surgimento dos TAs tem sido detectada no âmbito esportivo, sobretudo entre mulheres atletas de modalidades esportivas que preconizam restrita percentagem de gordura para aprimorar o rendimento e a estética corporal. Conjectura-se que a procura pela alta performance, demonstrada por pressões externas dos treinadores, patrocinadores, administradores e familiares na pretensão de cada vez mais apresentar os melhores resultados, ocasione estresse físico e mental aos jovens atletas, propiciando um ambiente para o desenvolvimento dos TAs (Fortes et al., 2014).

O balé clássico é uma modalidade de dança que aprecia a silhueta elegante e a estética corporal, e, posto isso, estimula seus praticantes à procura por um corpo ideal, desencadeando um descontentamento com a imagem corporal. Atrelado a isso vem o arquétipo de beleza exigido pela sociedade moderna, a busca incessante por uma melhor performance, e as pressões por parte dos instrutores, familiares, tornando também os bailarinos um grupo de risco para o desenvolvimento dos TA (Guimarães et al., 2014).

Inúmeros estudos demonstram a influência do ambiente familiar no estabelecimento do comportamento infantil e juvenil, e, por conseguinte, no desenvolvimento de seus transtornos, através dos quais a criança ou o adolescente tem uma apreensão exagerada com o peso e a forma do corpo (Gonçalves et al., 2013).

Existe uma relação positiva entre autoridade excessiva, aplicação de normas alimentares rígidas e individualização da alimentação com o surgimento dos TA (Gonçalves et al., 2013).

Vários estudos apontaram uma interiorização do ideal de beleza pela criança quando a mãe encorajava o emagrecimento, bem como, demonstraram uma relação positiva entre o hábito de assistir novelas, canais musicais e a restrição alimentar e insatisfação corporal entre pré-adolescentes do sexo feminino, antagonicamente existia uma relação negativa entre o hábito de assistir desenhos animados e a restrição alimentar (Gonçalves et al., 2013).

Alguns fatores atrelados ao surgimento dos transtornos são: o aumento no índice de massa corpórea (IMC); baixa autoestima; realização de dietas; medo da maturidade e insatisfação corporal (Gonçalves et al., 2013). 
Conquanto a busca pela magreza não esteja fortemente relacionada ao nível socioeconômico, a prevalência de comportamentos de risco tem sido encontrada em indivíduos nos estratos mais elevados (Gonçalves et al., 2013).

\section{Considerações finais}

Nota-se que independentemente da motivação á pratica de dietas restritivas, estas adquirem uma conotação de tolhimento, e no que tange a busca pelo "corpo ideal”, está de fato impossibilita e inviabiliza uma qualidade de vida satisfatória, através da supressão as leis que regem uma boa alimentação, atrelado a isso também está a supremacia da mídia que ludibria e induz as pessoas a esquecerem de si mesmas e a buscarem um estilo de vida restrito, solitário e opressor, mutilando egos e suscitando descontentamentos. Tais descontentamentos estão surgindo cada vez mais cedo, sobrepujando até mesmo os profissionais da área da saúde, trazendo consigo alterações cada vez mais significantes nos hábitos alimentares da população, culminando assim nos transtornos alimentares, avultando e diversificando cada vez mais seus grupos de risco, nesse contexto, necessita-se então de uma educação nutricional a longo prazo com abordagens inovadoras e conscientizadoras, visando desarreigar a pratica indiscriminada de dietas da moda e suas consequências na saúde dos seus adeptos.

\section{Referências}

Almeida, M. A. 2015. Transtorno Alimentar. Disponível em: http://www.psiclinicatcc.com.br/singlepost/2015/07/09/Transtorno-Alimentar. Acesso em: 3 de abr. 2018.

Almeida, A. P. de, Lima, F. M. V., Lisboa, S. M., Júnior, A. J. A. F., \& Lopes, A. P. 2013. Comparação entre as teorias da aprendizagem de Skinner e Bandura. Caderno De Graduação - Ciências Biológicas E Da Saúde UNIT, 1(3), 81-90.

Almeida, J. C., Rodrigues, T. C., Silva, F. M., \& Azevedo, M. J. 2009. Systematic review of weight loss diets: role of dietary components. Arquivos Brasileiros de Endocrinologia \& Metabologia, 53(5), 673-687.

Andreoli, C. S., Cortez, A. P. B., Sdepanian, V. L., \& Morais, M. B. 2013. Avaliação Nutricional e Consumo Alimentar de Pacientes com doença celíaca com e sem transgressão alimentar. Revista de Nutrição, 26(3), 301-11.

Baptista, T. J. R. 2013. A obesidade e a indústria do emagrecimento. ComCiência, 145, 0-0.

Betoni, F., Zanardo, V. P. S., \& Ceni, G. C. 2010. Avaliação de utilização de dietas da moda por pacientes de um ambulatório de especialidades em nutrição e suas implicações no metabolismo. ConScientiae Saúde, 9(3), 430-440.

Bechara, A. P. V., \& Kohatsu, L. N. 2014. Tratamento nutricional da anorexia e da bulimia nervosas: aspectos psicológicos dos pacientes, de suas famílias e das nutricionistas. Vínculo, 11(2), 07-18.

Bosi, M. L. M., Nogueira, J. A. D., Uchimura, K. Y., Luiz, R. R., \& Godoy, M. G. C. 2014. Eating behavior and body image among medicine students. Revista Brasileira de Educação Médica, 38(2), 243-252.

Carvalho, R. S., Amaral, A. C. S., \& Ferreira, M. E. C. 2009. Transtornos alimentares e imagem corporal na adolescência: uma análise da produção científica em psicologia. Psicologia: teoria e prática, 11(3), 200-223.

Cortez, C. M., Araújo, E. A., \& Ribeiro, M. V. 2011. Transtorno de compulsão alimentar periódico e obesidade. Arquivos Catarinenses de Medicina, 40(1), 94-102.

Damasceno, M. L. 2010. Transtornos Alimentares: Consequências da Busca do Corpo Magro e Desempenho Esportivo. Revista Hórus, 4(1), 254-271.

Escott-Stump, S. 2011. Nutrição relacionada ao diagnóstico e tratamento. 6.ed. São Paulo, SP: Manole, 1040p.

Fortes, L. S., Ferreira, M. E. C., Laus, M. F., \& Almeida, S. S. 2014. Insatisfação corporal e comportamento alimentar: comparações entre jovens atletas de diferentes esportes. Psicologia em Revista, 20(1),138-154.

Gonçalves, J. A., Moreira, E. A. M., Trindade, E. B. S. M., Fiates, G. M. R. 2013. Eating disorders in childhood and adolescence. Revista Paulista de Pediatria, 31(1), 96-103.

Guimarães, A. D., Machado, S. P., França, A. K. T. C., \& Calado, I. L. 2014. Transtornos alimentares e insatisfação com a imagem corporal em bailarinos. Revista Brasileira de Medicina do Esporte, 20(4), 267-271.

Kennedy, E. 2008. Putting the pyramid into action: the Healthy Eating Index and Food Quality Score. Asia Pacific Journal of Clinical Nutrition, 17, 70-74. 
Leonidas, C., \& Santos, M. A. 2015. Family relations in eating disorders: the Genogram as instrument of assessment. Ciência \& Saúde Coletiva, 20(5), 1435-1447.

Lima, E. S. 2009. Quantity, quality, harmony and adaption: the guiding principles of a society without hunger in Josué de Castro. História, Ciências, Saúde-Manguinhos, 16(1), 171-194.

Manuel, L. I. C. B. 2016. Perturbações alimentares, a ansiedade e a percepção da imagem corporal, em contexto académico. (Mestrado em Psicologia Clínica e da Saúde, Universidade Lusófona de Humanidades e Tecnologias, Escola de Psicologia e Ciências da Vida). Lisboa: ULHT.

Marangoni, J. S., \& Maniglia, F. P. 2017. Análise da composição nutricional de dietas da moda publicadas em revistas femininas. Revista da Associação Brasileira de Nutrição, 8(1), 31-36.

Mattar, R., \& Mazo, D. F. C. 2010. Intolerância à lactose: mudança de paradigmas com a biologia molecular. Revista da Associação Médica Brasileira, 56(2), 230-236.

Moore, K. 2014. Individual differences in restrained eaters. Tese apresentada para o Doutorado em Filosofia (Iowa State University). Ames, IO: ISU.

Oliveira, L. L., \& Hutz, C. S. 2010. Eating Disorders: the role of cultural aspects in the contemporary world. Psicologia em Estudo, 15(3), 575-582.

Ovbiosa-Akinbosoye, O. E., \& Long, D. A. 2011. Factors associated with long-term weight loss and weight maintenance: analysis of a comprehensive workplace wellness program. Journal of Occupational and Environmental Medicine, 53(11), 1236-1242.

Pereira, A. M. G. R. 2016. Preocupação com o peso e prática de dietas por adolescentes. Acta Portuguesa de Nutrição, 6, 14-18.

Previato, H., Volp, A. C. P., \& Freitas, R. 2014. Evaluation of diet quality by the Healthy Eating Index and its variations : A bibliographical review. Nutricion Clinica y Dietetica Hospitalaria, 34(2), 88-96.

Rola, M., \& Vasconcelos, C. 2014. Dieta Cetogénica - Abordagem Nutricional. Revista Nutrícias, 22, 16-19.

Santos, L. A. S. 2010. Da dieta à reeducação alimentar: algumas notas sobre o comer contemporâneo a partir dos programas de emagrecimento na Internet. Physis: Revista da Saúde Coletiva, 20(2), 459-474.

Silva, C. S. M., \& Kirsten, V. R. 2016. Avaliação da adequação de dietas publicadas por revistas não científicas. Revista Eletrônica Disciplinarum Scientia,15(1), 101-112.

Silva, J. D., Silva, A. B. J., Oliveira, A. V. K., \& Nemer, A. S. A. 2012. Influência do estado nutricional no risco para transtornos alimentares em estudantes de nutrição. Ciência \& Saúde Coletiva,17(12), 3399-3406.

Viana, D. S. O. 2014. Análise nutricional das dietas da moda. (Graduação em Nutrição, Centro Universitário de Brasília, Faculdade de Ciência da Educação e Saúde). Brasília, DF: UniCEUB.

Viana, L. V., Paula, T. P., Leitão, C. B., \& Azevedo, M. J. 2013. Fatores determinantes de perda de peso em adultos submetidos a intervenções dietoterápicas. Arquivos Brasileiros de Endocrinologia \& Metabologia, 57(9),717-721.

Vieira, C. M., Cordeiro, S. N., Magdaleno Júnior, R., \& Turato, E. R. 2011. Significados da dieta e mudanças de hábitos para portadores de doenças metabólicas crônicas: uma revisão. Ciência \& Saúde Coletiva, 16(7), 3161-3168.

\section{Minicurrículo}

Daniele Andreia Alvares. Nutricionista, formada pela Universidade Nove de Julho (2018); pós-graduada em Nutrição em Oncologia pela Faculdade Unyleya (2020); Pós-graduada em Abordagens Oncológicas na Área de Saúde pelo Centro Universitário São Camilo (2020); Pós-graduanda em Nutrição Clínica Avançada: Metabologia, Terapêutica Nutricional e Dietoterapia pela USCS (2020-2021); Mestranda Internacional em Ciências da Saúde (2020-2022). Áreas de maior interesse: Obesidade, Oncologia, Microbiota intestinal, DCNT.

Aline Alves de Santana. Possui graduação em Nutrição (2010) pela Universidade Nove de Julho, graduação em Formação Específica em Controle de Qualidade (2008) pela Universidade Nove de Julho. Extensão Universitária em Nutrição em Cardiologia (2011) pelo Instituto Dante Pazzanese de Cardiologia. Especialização em Obesidade, emagrecimento e saúde (2012) e Mestrado em Nutrição (2014) pela Universidade Federal de São Paulo. Possui experiência na área clínica-ambulatorial. Atualmente é professora da Universidade Nove de Julho na disciplina de Dietoterapia e Avaliação Nutricional e aluna de doutorado em 
Nutrição Experimental da Faculdade de Ciências Farmacêuticas da USP. Foi professora da graduação em Nutrição e Professora Visitante da Pós-graduação em Nutrição Clínica (Latu sensu) da Faculdade Integrada Coração de Jesus na disciplina de Bases da Nutrição Clínica (Bioquímica e Fisiopatologia da Nutrição). Áreas de maior interesse: Obesidade, compostos bioativos, inflamação, metabolismo lipídico.

Como citar: Alvares, D.A., \& Santana, A.A. Correlação entre dietas restritivas e o surgimento ou agravamento de transtornos alimentares. Pubsaúde, 4, a065. DOI: https://dx.doi.org/10.31533/pubsaude4.a065

Recebido: 18 ago. 2020.

Revisado e aceito: 25 ago. 2020.

Conflito de interesse: os autores declaram, em relação aos produtos e companhias descritos nesse artigo, não ter interesses associativos, comerciais, de propriedade ou financeiros que representem conflito de interesse.

Licenciamento: Este artigo é publicado na modalidade Acesso Aberto sob a licença Creative Commons Atribuição 4.0 (CC-BY 4.0). 